\title{
Single-beam multi-cantilever optical measurement head for cantilever array-based biosensors
}

\author{
Konrad Nieradka ${ }^{1}$, Daniel Kopiec ${ }^{1}$, Piotr Grabiec ${ }^{2}$, Maciej Węgrzecki ${ }^{2}$, Teodor Gotszalk ${ }^{1}$ \\ ${ }^{T}$ Faculty of Microsystem Electronics and Photonics, Wrocław University of Technology, \\ Janiszewskiego 11/17, 50-372 Wrocław, Poland \\ Corresponding author's e-mail address: konrad.nieradka@pwr.wroc.pl \\ ${ }^{2}$ Division of Silicon Microsystem and Nanostructure Technology, Institute of Electron Technology,
} Lotników 32/46, 02-668 Warsaw, Poland

\begin{abstract}
:
Here we present a homemade system for highly sensitive measurements of cantilever sensor arrays. Two unique techniques developed by our group are utilized. Firstly, the expanded beam deflection (EBD) method that allows truly simultaneous measurements of multiple cantilevers with a simple optical apparatus consisting of a single and stationary laser beam, while providing the same sensitivity as the original optical beam deflection (OBD) method. Secondly, the real-time Brownian noise extraction technique allows combined static-dynamic mode operation in gaseous or liquid environment with no need for external excitation of vibrations. Thus constructed measurement system allows highly sensitive simultaneous measurement of bending as well as flexural and torsional vibrations of up to 8 cantilevers.
\end{abstract}

Key words: cantilever sensor arrays, expanded beam deflection, Brownian noise, static mode, dynamic mode, static-dynamic mode

\section{Cantilever sensors}

Since invention of the atomic force microscope (AFM) by Binning et al. in 1986 [1] cantilever sensors are gaining increasing interest and wide applications, being constantly modified to perform new functions. In 1994 Gimzewski et al. employed a microcantilever as chemical reaction sensor, constructing first microcalorimeter [2]. The following years produced numerous publications on cantilever sensors and their various applications, summarized by Buchapudi et al. [3]. This yet young technology gained its recognition as a fast, versatile, and label-free analytical platform that can be applied to problems that other methods cannot solve or take too much effort, time, and money to solve.

\section{Optical detection methods}

The most sensitive method used to monitor motion of a micromechanical cantilever is the optical beam deflection (OBD) method, proposed by Meyer and Amer [4]. An optical beam is focused on the cantilever, reflected from its surface and directed onto a position sensitive detector. This method is used in the most of commercial and homemade AFM systems. It allows the cantilevers to be simpler and thinner than their piezoresistive counterparts allow and provides sensitivity equivalent to that of the interferometric method. It has been a challenge, though, to use this method on multicantilever arrays. Several approaches have been developed throughout recent years. They are schematically presented in Fig. 1.

Lang et al. [5] proposed using a timemultiplexed array of light sources in $1: 1$ projection onto the cantilever array and a single position sensitive detector. Álvarez et al. [6] proposed using a single light source to scan the cantilever array. The beam is reflected onto a single position sensitive detector and read out at previously determined intensity maxima. Both methods are quasi-simultaneous. A truly simultaneous readout system has been presented by Altmann et al. [7]. It consisted of two complete OBD setups aligned at an angle. It is, however, difficult to increase the number of cantilevers read out using this method.

\section{Expanded beam deflection method}

The EBD method, proposed by our group [8], allows simultaneous measurement of multiple cantilevers without time multiplexing and without multiplication of optical paths, while maintaining sensitivity of the OBD method. Its 
concept is shown in Fig. 1. The detector type depends on the mode of sensing. For dynamic mode operation, dedicated photodiode arrays are used to maximize sensitivity, while for static and combined static-dynamic mode operation, a specialized lateral-effect sensor arrays are used to ensure linear response characteristics.

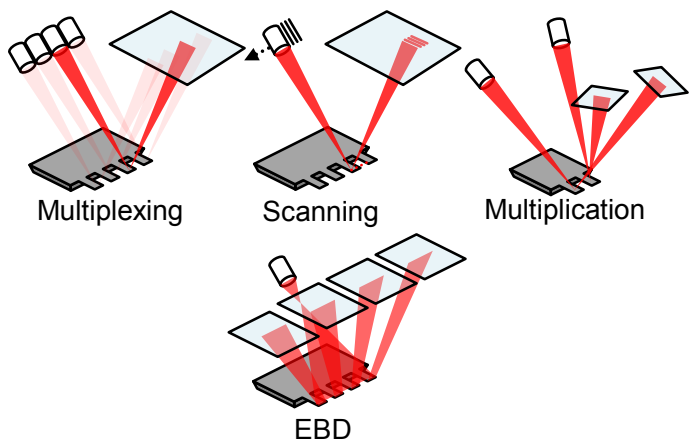

Fig. 1. Overview of multicantilever $O B D$ technique implementations: multiplexing - multiple timemultiplexed light sources and a common detector; scanning - single, scanning light source and a single detector; multiplication - multiple complete $O B D$ paths aligned at an angle; EBD - single, stationary light source and multiple detectors.

\section{Brownian noise extraction technique}

In dynamic mode sensing, no external excitation of vibrations is used. Instead, a realtime Brownian noise extraction technique is used [9]. A fast Fourier transform (FFT) is performed on the system's intrinsic noise and the spectra are averaged multiple times, revealing Brownian noise resonance peaks. The resonance frequencies and other parameters are extracted by fitting Lorentz curves to selected peaks using LevenbergMarquardt algorithm.

\section{MCAS system}

Our Micro Cantilever Array Sensors measurement system (MCAS) consists of 7 distinctive parts, marked on a photography in Fig. 2. The laser module encompasses a constant-power laser controller, a radiator, a collimator, and a pair of cylindrical lenses. It is mounted in the optical head equipped with two tilting mirrors and two translation stages, which allow the laser beam to be positioned on the cantilever array and forwarded onto the detector.

The detector itself may have a form of a lateraleffect photodiode array or a classical photodiode array. For the former we use commercially available 16-element 1-D PSD array (1LA16-2,5_SU89, Sitek / Laser Components), while for the latter we have fabricated our own $8 \times 2$ photodiode arrays. The detector is connected with the module of 32 current-to-voltage converters. Converted signals are fed to the analog arithmetic circuits, which perform addition, subtraction, and division operations that depend on the actual detector configuration.

The base of the system forms the suspension for the flow cell. It allows easy replacement of flow cells and additional tilt correction as a compensation for varying refractive indices of used media. Finally, the flow cell made of polyether ether ketone (PEEK) is mounted in the suspension. It has a pair of ports for supplying gas or liquid, top and bottom window for optical access, and a removable cantilever holder. It has also one additional port for optional reference electrode, which allows electrochemical measurements. In such case, the cantilever serves as the working electrode and the counter electrode is integrated with the cantilever holder.
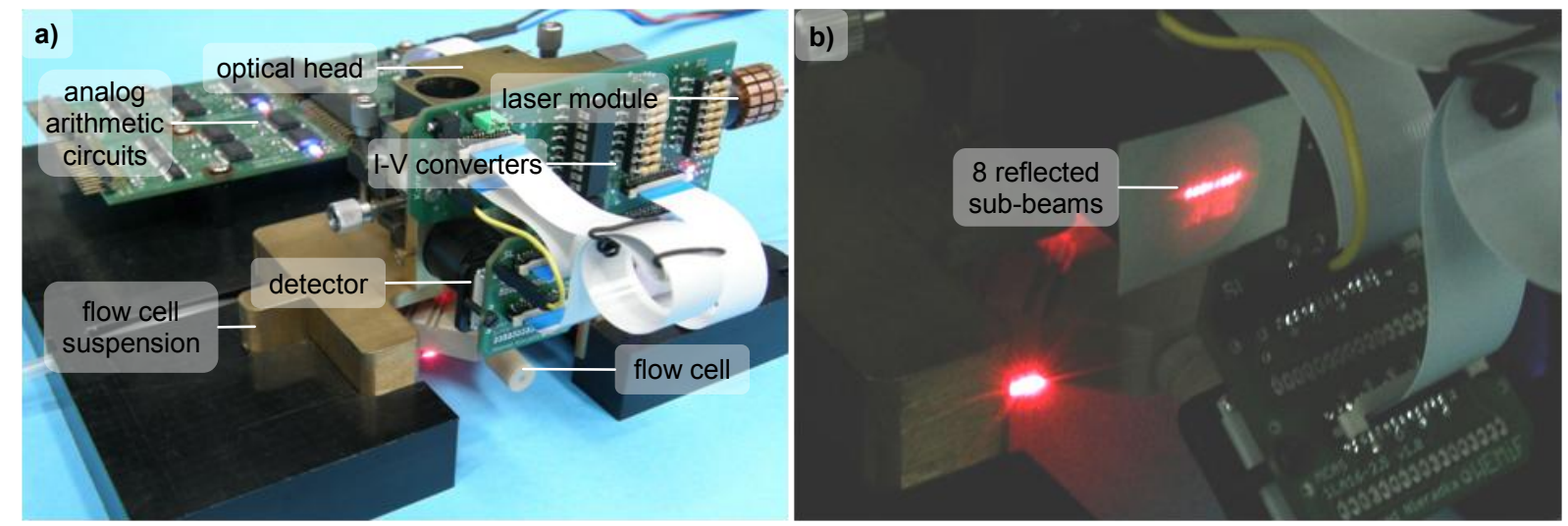

Fig. 2. Complete hardware photography: a) distinctive parts of the MCAS system; b) a paper sheet slid in place of the detector to reveal 8 sub-beams reflected from an 8-cantilever array (Octisensis, Micromotive Mikrotechnik). The intensity of reflected beams is reduced due to lack of gold coating on the cantilevers and their $1 \mu m$ thickness which make them almost transparent. 
A paper sheet slid in place of the detector in Fig. $2 b$ reveals 8 sub-beams reflected from 8-cantilever array.

\section{Cantilever arrays}

We have used three types of cantilever arrays for our experiments. We have fabricated our own 4-cantilever arrays (designated ITE). We have also used commercial 2-cantilever arrays (Arrow TL2Au, Nanoworld) and 8-cantilever arrays (Octisensis, Micromotive Mikrotechnik). In each case the length of the cantilevers was $500 \mu \mathrm{m}$, the width was $100 \mu \mathrm{m}$, and the pitch was $250 \mu \mathrm{m}$. ITE cantilevers were $2 \mu \mathrm{m}$ thick and TL2Au and Octisensis were $1 \mu \mathrm{m}$ thick. Top side of the ITE and TL2Au cantilevers was coated with gold.

\section{Results and discussion}

Fig. 3 shows acquired thermal noise spectra of ITE array. It demonstrates thermal noise resolution of the system even for stiffer cantilevers ( $1^{\text {st }}$ eigenmode at $33.4 \mathrm{kHz}$ ). No crosstalk between resonance peaks is observed.

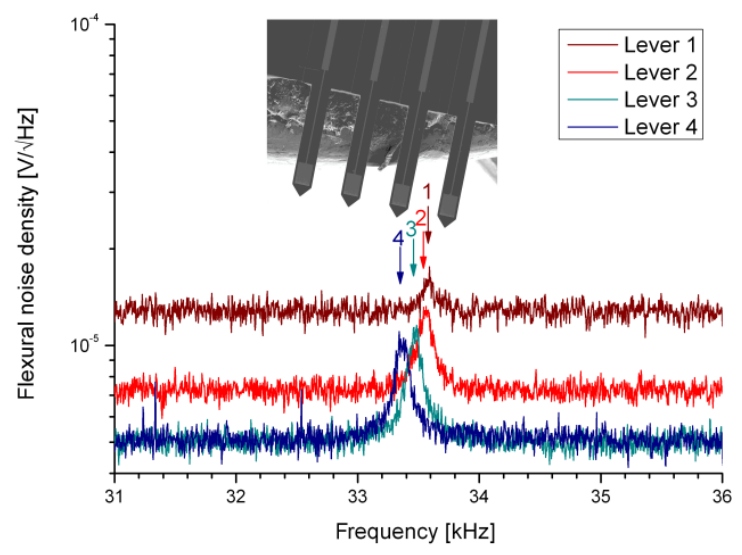

Fig. 3. Thermal noise of a microcantilever array measured in air in the MCAS system. Four ITE microcantilevers have been measured simultaneously using homemade $8 \times 2$-section photodiode. The photo-inset shows a SEM image of the cantilever array used.

In Fig. 4 we present similar measurement, though performed in water using softer TL2Au array and lateral effect photodiode array. The thermal noise resolution is also achieved, clearly revealing three first flexural modes. In the band between $2^{\text {nd }}$ and $3^{\text {rd }}$ flexural mode one may even see a minute geometrical cross-talk from $1^{\text {st }}$ torsional mode.

Having thermal resolution combined with linear characteristic of lateral effect photodiode allows operating our sensors in combined staticdynamic mode.

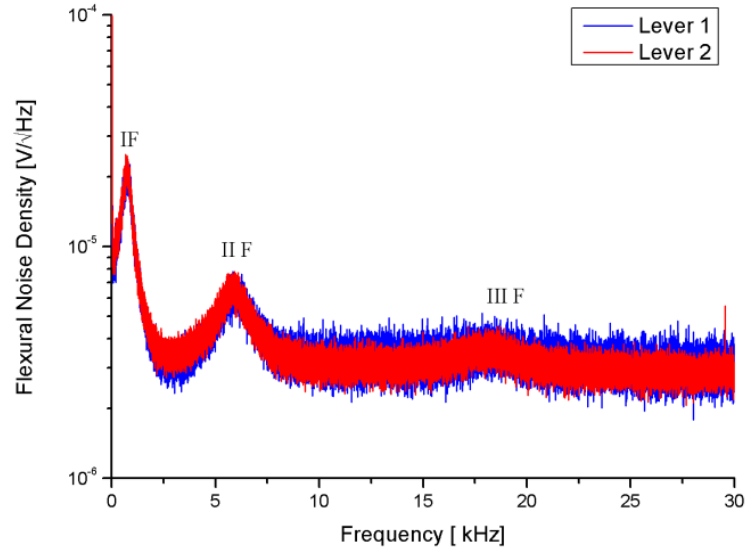

Fig. 4. Thermal noise of a microcantilever array measured in liquid in the MCAS system. Two TL2Au cantilevers have been measured simultaneously using lateral effect photodiode array. The three first flexural resonances shown in this plot overlap each other closely.

An example of measurement performed in such combined mode is shown in Fig. 5. TL2Au array has been differentially functionalized to respond differently for humidity. Left cantilever was made hydrophilic using 11-mercaptoundecanoic acid and right cantilever was made hydrophobic using 1-undecanethiol. Both cantilevers were measured simultaneously while different mixtures of dry and humid nitrogen were pumped through the flow cell.

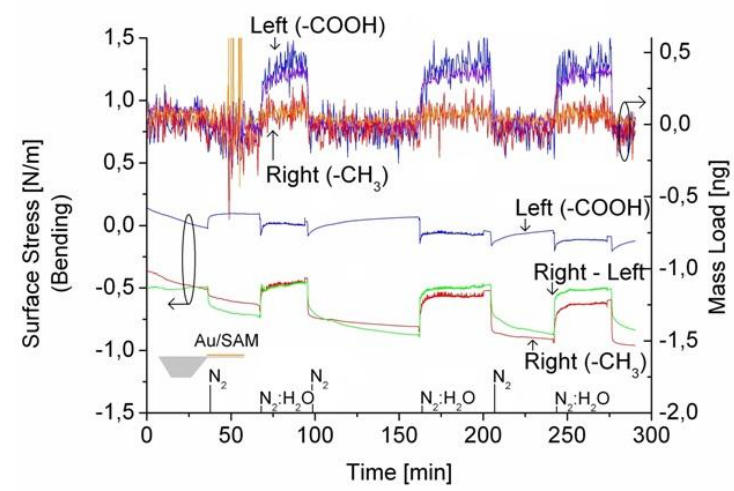

Fig. 5. Environmental factors measurement in combined static-dynamic mode using differentially functionalized TL2Au microcantilever array. The gold side of left cantilever has been coated with hydrophilic 11-mercaptoundecanoic acid SAM while that of right cantilever has been coated with hydrophobic 1-undecanethiol SAM. The native silicon oxide on the opposite side of the cantilevers was left unmodified, yielding minute hydrophilicity. The upper plots show added mass calculated from resonance frequency shift for the $1^{\text {st }}$ and $2^{\text {nd }}$ flexural mode. The lower plots show the bending of the microcantilevers and the differential signal.

The hydrophobic cantilever undergoes only slight mass loading (below $100 \mathrm{pg}$ ) in reaction to moisture due to minute hydrophilicity of the bottom silicon oxide side. The hydrophilic 
cantilever is loaded with almost $500 \mathrm{pg}$ of water in response to humid nitrogen.

Since both sides of hydrophilic cantilever adsorb a layer of water, the differential surface stress is low. In the case of hydrophobic cantilever, we observe higher values of differential surface stress. After subtraction of static signals of both cantilevers, the initial thermal drift is compensated and signal with clear baseline is acquired, carrying information on the hydrophilic cantilevers gold side surface stress.

\section{Conclusions}

In our contribution, we have presented the design and operational characteristics of cantilever array optical measurement system along with comparison to other solutions present in the literature. The system is characterized by $250 \mathrm{fm} / \sqrt{ } \mathrm{Hz}$ noise floor in all channels for photodiode array and thermal noise resolution for lateral-effect sensor arrays. We have shown preliminary measurements in gaseous and liquid environments using cantilevers functionalized with self-assembling monolayers (SAMs).

\section{Acknowledgements}

This paper has been written as a result of realization of the project entitled: "Micro- and Nanosystems in Chemistry and Biomedical Diagnostics“. The project co-financed by the European Union via the European Regional Development Fund and the Polish state budget, within the framework of the Operational Programme Innovative Economy 2007-2013. Contract no. POIG.01.03.01-00-014/08

\section{References}

[1] G. Binnig, C.F. Quate, C. Gerber, Atomic Force Microscope, Physical Review Letters 56, 930933 (1986); doi: 10.1103/PhysRevLett.56.930

[2] J.K. Gimzewski, C. Gerber, E. Meyer, R.R. Schlittler, Observation of a chemical reaction using a micromechanical sensor, Chemical Physics Letters 217, 589-594 (1994); doi: 10.1016/0009-2614(93)E1419-H

[3] K.R. Buchapudi, X. Huang, X. Yang, H.F. Ji, T. Thundat, Microcantilever biosensors for chemicals and bioorganisms, Analyst 136, 15391556 (2011); doi: 10.1039/COAN01007C

[4] G. Meyer, N. M. Amer, Novel optical approach to atomic force microscopy, Applied Physics Letters 53, 1045-1047 (1988); doi: 10.1063/1.100061

[5] H. P. Lang, R. Berger, F. Battiston, J.-P. Ramseyer, E. Meyer, C. Andreoli, J. Brugger, P. Vettiger, M. Despont, T. Mezzacasa, L. Scandella, H.-J. Güntherodt, Ch. Gerber, J. K. Gimzewski, A chemical sensor based on a micromechanical cantilever array for the identification of gases and vapors, Applied Physics A 66, 61-64 (1998); doi: $10.1007 / \mathrm{s} 003390051100$

[6] M. Álvarez, J. Tamayo, Optical sequential readout of microcantilever arrays for biological detection, Sensors and Actuators B 106, 687-690 (2005); doi: 10.1016/j.snb.2004.09.016

[7] S. M. Altmann, P.-F. Lenne, and J.-K. H. Hörber, Multiple sensor stabilization system for local probe microscopes, Review of Scientific Instruments 72, 142-149 (2001); doi: 10.1063/1.1333044

[8] K. Nieradka, G. Małozięć, D. Kopiec, P. Grabiec, P. Janus, A. Sierakowski, T. Gotszalk, Expanded beam deflection method for simultaneous measurement of displacement and vibrations of multiple microcantilevers, Review of Scientific Instruments 82, 105112 (2011); doi: 10.1063/1.3652977

[9] K. Nieradka, T. Gotszalk, G. Schroeder, A novel method for simultaneous readout of static bending and multimode resonance-frequency of microcantilever-based biochemical sensors, Sensors and Actuators B (2010); in press; doi: 10.1016/j.snb.2011.05.032 